\title{
Can the gamma-ray bursts travelling through the interstellar space be explained without invoking the drastic assumption of Lorentz invariance violation?
}

\author{
M. Chaichian, ${ }^{a, *}$ I. Brevik ${ }^{b}$ and M. Oksanen ${ }^{a}$ \\ ${ }^{a}$ Department of Physics, University of Helsinki, \\ P.O.Box 64, FI-00014 Helsinki, Finland \\ ${ }^{b}$ Department of Energy and Process Engineering, Norwegian University of Science and Technology, \\ N-7491 Trondheim, Norway \\ E-mail: masud.chaichian@helsinki.fi, iver.h.brevik@ntnu.no, \\ markku . oksanen@helsinki . fi
}

Experimental observations indicate that gamma-ray bursts (GRB) and high-energy neutrino bursts may travel at different speeds with a typical delay measured at the order of hours or days. We discuss two potential interpretations for the GRB delay: dispersion of light in interstellar medium and violation of Lorentz invariance due to quantum gravitational fluctuations. Among a few other media, we consider dispersion of light in an axion plasma, obtaining the axion plasma frequency and the dispersion relation from quantum field theory for the first time. We find that the density of axions inferred from observations is far too low to produce the observed GRB delay. However, a more precise estimation of the spatial distribution of axions is required for a conclusive result. Other known media are also unable to account for the GRB delay, although there remains uncertainties in the observations of the delays. The interpretation in terms of Lorentz invariance violation and modified dispersion relation suffers from its own problems: since the modification of the dispersion relation should not be dependent on particle type, delays between photons and neutrinos are hard to explain. Thus neither interpretation is sufficient to explain the observations. We conclude that a crucial difference between the two interpretations is the frequency dependence of the propagation speed of radiation: in dispersive plasma the group speed increases with higher frequency, while Lorentz invariance violation implies lower speed at higher frequency. Future experiments shall resolve which one of the two frequency dependencies of GRB is actually the case.

40th International Conference on High Energy physics - ICHEP2020

July 28 - August 6, 2020

Prague, Czech Republic (virtual meeting)

\footnotetext{
*Speaker
} 


\section{Introduction}

Gamma-ray bursts (GRB) are highly energetic and diverse events, which are thought to be produced by violent stellar processes, in particular supernovas and mergers of binary neutron stars. Those events may also produce high-energy cosmic rays and consequently bursts of high-energy neutrinos [1]. Neutrino bursts have been observed to be shifted in time with respect to the GRB (see $[2,3]$ and references therein). The time window $\tau=t_{G R B}-t_{v}$ between the arrival times of a GRB $t_{G R B}$ and a neutrino burst $t_{v}$ can vary between an hour or several days. Assuming that a GRB and the corresponding neutrino burst are produced at the same time or within a short period, a significant delay $\tau$ would indicate that the electromagnetic and neutrino signals have travelled at different speeds. Note, however, that the recent experimental studies show only faint neutrino signals associated with GRB [2,3], and hence the observed delays may be inaccurate.

It is equally challenging to interpret the GRB delay within standard physics where GRB are delayed due to the interaction of photons with interstellar media, a phenomenon which always occurs. In this way, one can also shed additional light on the "microstructure" of the Universe or a part of it and its constituents. The interaction of neutrinos with any interstellar medium is extremely weak and hence the dispersion of neutrinos is negligible. Secondly, while the neutrinos are massive and oscillating, the effect on the speed of high-energy neutrinos is very small. Consider a GRB with photon energy $1 \mathrm{TeV}$ and neutrinos with the same energy, $E=1 \mathrm{TeV}$. The dispersion relation $E^{2}=p^{2} c^{2}+m^{2} c^{4}$ gives the speed of the neutrinos as $v_{v}=\frac{d E}{d p} \approx c\left(1-d_{v}\right)$, where $d_{v}=\frac{m^{2} c^{4}}{2 E^{2}}$. Averaging over 3 neutrinos, $\left\langle m^{2} c^{4}\right\rangle=(1 / 3)(0.1 \mathrm{eV})^{2}$, where the masses are estimated with the heaviest neutrino mass. The speed of neutrinos is given by $d_{v}=0.17 \times 10^{-26}$. Thus the delay compared to a signal travelling at the speed $c$ would be measured in nanoseconds even for signals from furthest galaxies: $\tau=D \times d_{v} / c \lesssim 10^{-8} \mathrm{~s}$, using a maximal travelling distance $D=10^{27} \mathrm{~m}$ (across the whole universe). That is negligible compared to the observed GRB delays. Theories of neutrino production in GRB actually predict neutrinos with even higher energy of order $10^{2}-10^{7}$ $\mathrm{TeV}$ [1], which means $v_{v}$ is even closer to $c$. This justifies $v_{v}=c$ in our estimates.

Violation of Lorentz invariance and the associated modification of the dispersion relation has been considered as a potential interpretation of the delay of high-energy GRB [4,5]. This approach is motivated by various approaches to quantum gravity, since quantum-gravitational fluctuations may lead to a non-trivial refractive index [6]. We shall comment the Lorentz invariance violation interpretation in Sec. 4.

Before seeking to modify fundamental principles such as Lorentz invariance we prefer to consider possible explanations for the observed phenomena by means of standard physics. We consider the dispersion of light in several media and assess the produced GRB delay when photons and neutrinos are assumed to be emitted from the same source at the same time. Neither electron plasma nor photon plasma can account for the observed GRB delay. Then we consider axions. Axions are pseudoscalar particles that may both provide a solution to the strong $\mathrm{CP}$ problem and constitute cold dark matter. Axions are not electrically charged, since a charged axion would be luminuous, but can still interact with photons. Axion electrodynamics has been studied actively and it is connected to topological insulators [7, 8]. Therefore an axion plasma is a plausible cosmic medium that would have an effect on the propagation of light from distant galaxies. We derive the dispersion relation in an axion plasma and assess its effect on the GRB delay. 


\section{Dispersion relation and plasma frequency}

A plasma can support both longitudinal and transverse waves. We are interested in transverse waves. Dispersion relation for light in a plasma is

$$
\omega^{2}=c^{2} \boldsymbol{k}^{2}+\omega_{p}^{2}
$$

where $\omega_{p}$ is the plasma frequency. The angular frequency is also given as $\omega=\boldsymbol{v}(\hat{\boldsymbol{k}}) \cdot \boldsymbol{k}=\frac{c|\boldsymbol{k}|}{n}$, where $n$ is the refraction index and $\boldsymbol{v}=\frac{c}{n} \hat{\boldsymbol{k}}$ is the phase velocity, where $\hat{\boldsymbol{k}}=\frac{\boldsymbol{k}}{|\boldsymbol{k}|}$. Thus the refraction index is related to the plasma frequency as

$$
n^{2}=1-\frac{\omega_{p}^{2}}{\omega^{2}}
$$

In an isotropic medium, $\omega=\omega(|\boldsymbol{k}|)$ and $\omega_{p}=\omega_{p}(|\boldsymbol{k}|)$, group velocity is parallel to phase velocity. When the photon momentum is large compared to the plasma frequency, $c^{2} \boldsymbol{k}^{2} \gg \omega_{p}^{2}$, we obtain that group velocity is only slightly lower than $c$,

$$
v_{g}=\frac{\partial \omega(|\boldsymbol{k}|)}{\partial|\boldsymbol{k}|} \simeq c\left(1-\frac{\omega_{p}^{2}}{2 \omega^{2}}\right) \equiv c(1-d) .
$$

Now we explain how the plasma frequency and refraction index can be derived from quantum field theory. From here on we assume units $\hbar=c=\epsilon_{0}=1$. The refraction index is related to the forward scattering amplitude $f(0)$ as [9]

$$
n=1+2 \pi \frac{N f(0)}{\omega^{2}}
$$

where $N$ is the number density of scatterers. The relation (4) is valid when $n$ is close to one, $|n-1| \ll 1$, and follows from the inteference between incident and scattered waves. Inserting (2) into (4), we obtain a relation between the plasma frequency and the scattering amplitude. When the photon frequency is large compared to the plasma frequency, $\omega^{2} \gg \omega_{p}^{2}$, the relation is given as

$$
\omega_{p}^{2}=-4 \pi N f(0) .
$$

The scattering amplitude $f(\theta)$ is defined as a part of the wavefunction at large distance $r$ from the scatterer, $\psi(\boldsymbol{r})=C\left(e^{i \boldsymbol{k} \cdot \boldsymbol{r}}+f(\theta) e^{i k r} / r\right)$, where $C$ is a normalization factor. The differential cross section is given in terms of the scattering amplitude as $d \sigma(\theta)=|f(\theta)|^{2} d \Omega$. The differential cross section can as well be obtained from quantum field theory. Hence we obtain the differential cross section $d \sigma$ at angle $\theta=0$ in quantum field theory and identify the forward scattering amplitude as

$$
|f(0)|=\left(\frac{d \sigma(0)}{d \Omega}\right)^{\frac{1}{2}} .
$$

For an electron plasma, we obtain the differential cross section for scattering of a photon on an electron in quantum field theory. In the rest frame of the initial electron, we obtain $d \sigma(0)=$ $\left(e^{4} / 16 \pi^{2} m_{e}^{2}\right) d \Omega$, and according to (6) we get $|f(0)|=\alpha / m_{e}$, where $\alpha$ is the fine-structure constant, $\alpha=e^{2} / 4 \pi$, and $m_{e}$ is the electron mass. Thus the plasma frequency (5) is given as

$$
\omega_{p}^{2}=\frac{N e^{2}}{m_{e}},
$$


which is the same result that is obtained from classical electrodynamics [10,11]. With $m_{e}=$ $0.511 \mathrm{MeV}$, we obtain the group velocity (3) for the photon energy $1 \mathrm{TeV}$,

$$
v_{G R B}=c\left(1-0.7 \times 10^{-51} \times N \times \text { metre }^{3}\right) .
$$

Therefore, dispersive properties of an electron gas are not significant enough to account for a time delay of the order of several hours as observed.

Dispersion of light in light plasma also produces a too small delay. We obtain from light on light scattering $\omega_{p}^{2}=$ const. $\times N_{\gamma} e^{4} / \omega$, where $N_{\gamma}$ is the number density of photons. For a delay of the order of few hours, we would need photon density $N_{\gamma}=10^{39} \mathrm{~m}^{-3}$, while according to the Planck data on the CMB (Cosmic Microwave Background) radiation: $N_{\gamma}=(4-5) \times 10^{8} \mathrm{~m}^{-3}$.

\section{Axion plasma and its effect on the propagation of gamma-rays}

Interaction Lagrangian of axion electrodynamics is [12]

$$
\mathcal{L}_{a \gamma \gamma}=-\frac{1}{4} g a F_{\mu \nu} \tilde{F}^{\mu \nu}=-\frac{1}{2} g a \epsilon^{\mu \nu \rho \sigma} \partial_{\mu} A_{\nu} \partial_{\rho} A_{\sigma},
$$

where $g$ is a coupling constant, $a$ is the axion pseudoscalar field, the electromagnetic field strength tensor is $F_{\mu \nu}=\partial_{\mu} A_{\nu}-\partial_{\nu} A_{\mu}$, and its dual $\tilde{F}^{\mu \nu}=\frac{1}{2} \epsilon^{\mu \nu \rho \sigma} F_{\rho \sigma}$.

Consider scattering of photon on axion $\gamma+a \rightarrow \gamma+a$ at tree level. The scattering amplitude $\mathcal{M}$ is a sum of two terms represented by the diagrams in Fig. 1. We are interested in scattering with
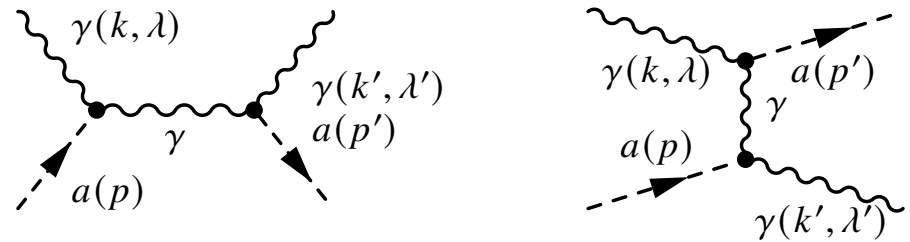

Figure 1: Feynman diagrams for scattering of photon on axion (drawn from left to right)

parallel momenta for initial and final photons, i.e. with angle $\theta=0$. The differential cross section for unpolarized photons with angle $\theta=0$ is obtained in the rest frame of the initial axion as

$$
d \sigma(0)=\frac{1}{64 \pi^{2} m_{a}^{2}}\left(\frac{1}{2} \sum_{\lambda, \lambda^{\prime}}|\mathcal{M}(0)|^{2}\right) d \Omega=\left(\frac{3 g^{2}}{16 \pi}\right)^{2}\left|\frac{\omega^{2}}{\left(2 \omega+m_{a}\right)}+\frac{\omega^{2}}{\left(-2 \omega+m_{a}\right)}\right|^{2} d \Omega .
$$

where $\omega$ is the energy of the initial photon. The scattering amplitude $|f(0)|$ is obtained according to (6). Since the axions are very light, $m_{a} \sim 10^{-5} \mathrm{eV}$, and we are interested in very high energy photons, we consider the limit $\omega \gg m_{a}$. For photons with energies well above the axion mass, the plasma frequency is nearly constant, i.e. independent of the frequency of the incoming light:

$$
\omega_{p}^{2}=\frac{3}{8} N g^{2} m_{a}\left(1+\frac{m_{a}^{2}}{4 \omega^{2}}+O\left(\frac{m_{a}^{4}}{\omega^{4}}\right)\right) \simeq \frac{3}{8} N g^{2} m_{a} .
$$

Estimating the effective coupling constant to be $g=10^{-10} \mathrm{GeV}^{-1}$, and the axion mass $m_{a}=$ $10^{-5} \mathrm{eV}$, we obtain the group velocity for the photon of energy $1 \mathrm{TeV}$ in an axion plasma,

$$
v_{G R B}=c(1-d), \quad d=\frac{3}{16} \frac{N g^{2} m_{a}}{\omega^{2}}=1.4 \times 10^{-88} \times N \times \text { metre }^{3} .
$$


Thus the delay of the GRB in the galactic plasma is

$$
\tau=\frac{D \times d}{c}=\frac{3}{16} \frac{N g^{2} m_{a}}{\omega^{2}} \frac{D}{c}=4.8 \times 10^{-97} \times D \times N \times \text { metre }^{2} \times \text { second },
$$

where $D$ is the distance traveled by the photons. Typical value of the delay $\tau$ taken from ANTARES data is $\tau=3.25$ hours. The effective distance travelled by photons in expanding Universe depends on the redshift $z$ [2], $D(z)=\frac{c}{H_{0}} \int_{0}^{z} \frac{(1+z) d z}{\sqrt{\Omega_{m}(1+z)^{3}+\Omega_{\Lambda}}}$. If $D$ is taken as the diameter of observable Universe, $D=8.8 \times 10^{26} \mathrm{~m}$, we need an axion number density $N \simeq 10^{73} \mathrm{~m}^{-3}$. This is a very large number density that apparently contradicts experimental data.

A more realistic scenario is to consider that axions are concentrated in galactic halos (constituting cold dark matter). The mass density of axions in a galactic halo is estimated $D_{m}=$ $0.45 \mathrm{GeV} / \mathrm{cm}^{3}$, and the radius of the halo is $5 \times 10^{20} \mathrm{~m}$ [14]. Number density of axions is $N_{G H}=0.45 \times 10^{20} \mathrm{~m}^{-3}$. In order to produce the delay $\tau=3.25$ hours, the axion number density in galactic halo should be $N \simeq 10^{79} \mathrm{~m}^{-3}$, which is much higher than from data $N_{G H}$ multiplied by any number of farther galaxies within the diameter of the Universe.

Details of the derivation of (11) will be presented elsewhere [13]. For related works on the bending of light in axion backgrounds but not considering the issues concerning GRB, see [15] and references therein.

\section{Resolution between dispersion in plasma and Lorentz invariance violation}

In the quantum gravity motivated interpretation that violates Lorentz invariance $[4,5]$, the dispersion relation is modified to contain higher-power energy terms (or higher-power momentum terms), $E^{2}\left[1+\sum_{n=1} \xi_{n}\left(E / E_{Q G}\right)^{n}\right]=p^{2} c^{2}+m^{2} c^{4}$. Then the group velocity of light is

$$
v_{g}=c\left(1-\xi \frac{E}{E_{Q G}}+O\left(\frac{E^{2}}{E_{Q G}^{2}}\right)\right),
$$

where $E_{Q G}$ is an effective quantum gravity energy scale, usually of order $E_{Q G}=10^{16} \mathrm{GeV}$. Hence the modification of the dispersion relation implies that the slowdown of radiation is increased with higher energy. Thus this approach has mainly been used to consider the delay between higher energy photons and lower energy photons produced in GRB. A delay between neutrinos and photons produced in GRB might be possible in this interpretation only if the energy of neutrinos is several orders of magnitude higher than the energy of photons [5].

The key feature that differentiates the dispersive plasma interpretation from the Lorentz invariance violation (LIV) interpretation is the energy dependence of the signal delay. In plasma the delay decreases with higher photon frequency, $\tau \propto \omega^{-2}$, while in the LIV case it increases with frequency, $\tau \propto \omega$. It would be crucial to test the frequency/energy dependence of the delay experimentally. That requires the exact measurement time of observation of GRB and spectral resolutions and therefore, the planned broad energy range measurements are utmost crucial [16].

Since neither LIV nor dispersion of light in a plasma can explain such a large delay between GRB and neutrinos, one could even suspect the existence of the delay with such an amount. 


\section{References}

[1] E. Waxman and J. N. Bahcall, High-energy neutrinos from cosmological gamma-ray burst fireballs, Phys. Rev. Lett. 78 (1997) 2292 [astro-ph/9701231].

[2] S. Adrián-Martínez et al. [ANTARES], Stacked search for time shifted high energy neutrinos from gamma ray bursts with the ANTARES neutrino telescope, Eur. Phys. J. C 77 (2017) 20 [arXiv: 1608.08840 [astro-ph.HE]].

[3] M. G. Aartsen et al. IceCube Search for High-Energy Neutrino Emission from TeV Pulsar Wind Nebulae, Astrophys. J. 898 (2020) 117 [arXiv: 2003.12071 [astro-ph.HE]].

[4] G. Amelino-Camelia, J. R. Ellis, N. E. Mavromatos, D. V. Nanopoulos and S. Sarkar, Tests of quantum gravity from observations of gamma-ray bursts, Nature 393 (1998) 763 [astro-ph/9712103].

[5] U. Jacob and T. Piran, Neutrinos from gamma-ray bursts as a tool to explore quantum-gravityinduced Lorentz violation, Nature Phys. 3 (2007) 87 [hep-ph/0607145].

[6] J. R. Ellis, N. E. Mavromatos and D. V. Nanopoulos, Derivation of a vacuum refractive index in a stringy space-time foam model, Phys. Lett. B 665 (2008) 412 [arXiv: 0804 . 3566 [hep-th]].

[7] F. Wilczek, Two Applications of Axion Electrodynamics, Phys. Rev. Lett. 58 (1987) 1799.

[8] A. Martín-Ruiz, M. Cambiaso and L. F. Urrutia, Green's function approach to Chern-Simons extended electrodynamics: An effective theory describing topological insulators, Phys. Rev. D 92 (2015) 125015 [arXiv: 1511.01170 [cond-mat. other]].

[9] N. Bohr, R. Peierls and G. Placzek, Nuclear Reactions in the Continuous Energy Region, Nature 144, 200 (1939).

[10] M. Chaichian, I. Merches, D. Radu and A. Tureanu, Electrodynamics: An Intensive Course, Springer-Verlag, 2016.

[11] J.D. Jackson, Classical electrodynamics, 3rd Edition, John Wiley \& Sons, 1999.

[12] P. Sikivie, Invisible axion search methods, arXiv:2003.02206 [hep-ph].

[13] I. Brevik, M. Chaichian and M. Oksanen, to appear.

[14] T. Braine et al. [ADMX Collaboration], Extended search for the invisible axion with the axion dark matter experiment, Phys. Rev. Lett. 124 (2020) 101303 [arXiv:1910.08638 [hep-ex]].

[15] J. I. McDonald and L. B. Ventura, Bending of light in axion backgrounds, arXiv : 2008.12923 [hep-ph].

[16] V. A. Acciari et al. [MAGIC], Observation of inverse Compton emission from a long $\gamma$-ray burst, Nature 575 (2019) 459 [arXiv: 2006.07251 [astro-ph.HE]]. 